\title{
artigo
}

Capelasso, C.S.; Garbugio, L.L.; Marques, F.R.D.M.; Pires, G.A.R.; Salci, M.A.; Charlo, P.B.;

Grau de dependência de nicotina no programa de controle do tabagismo em Maringá: estudo de caso

\section{Grau de dependência de nicotina no programa de controle do tabagismo em Maringá: estudo de caso}

\author{
Degree of nicotine dependence in the smoking control program in Maringá: case study
}

Grado de dependencia a la nicotina en el programa de control de fumadores en Maringá: estudio de caso

\begin{abstract}
RESUMO
Objetivo: Identificar dependência nicotínica em usuários que não cessaram o tabagismo após o Programa de Controle do Tabagismo na unidade de saúde selo ouro. Método: Estudo de caso de natureza quantitativa, realizado na Unidade Básica de Saúde selo ouro de Maringá-PR, em 2020. Participaram usuários que compareceram em todas sessões do programa em 2018 e 2019, e que não cessaram o tabagismo após intervenção. Para coleta, utilizou-se entrevista guiada por instrumento estruturado. Organizou-se os dados pelo Microsoft Excel 2016 e analisou-se estatística descritivamente simples. Respeitando todos os preceitos éticos. Resultados: Houveram 17 participantes, 64,7\% mulheres, 58,8\% acima dos 60 anos, 35,3\% casados, 47,05\% baixa escolaridade e 29,4\% com elevada dependência nicotínica. Não houve significância associativa entre variáveis demográficas e grau de dependência. Conclusão: 0 grau elevado de dependência nicotínica em participantes do programa pode auxiliar ações para cessação tabágica favorecendo estratégias para ampliar a participação nos grupos de controle tabágico.
\end{abstract}

DESCRITORES: Prevenção do Hábito de Fumar; Tabagismo; Abandono do Uso de Tabaco.

\section{ABSTRACT}

Objective: To identify nicotine dependence in users who did not stop smoking after the Smoking Control Program in a Gold Seal Health Unit. Method: Quantitative case study, carried out in a Gold Seal Basic Health Unit of Maringá-PR, in 2020. The participants were users who attended all sessions of the program in 2018 and 2019, who did not stop smoking after the intervention. For collection, we used an interview guided by a structured instrument. The data was organized by Microsoft Excel 2016 and descriptively simple statistics were analyzed. All ethical precepts were respected. Results: 17 people participated in the study, $64.7 \%$ female, $58.8 \%$ over 60 years of age, $35.3 \%$ married, $47.05 \%$ with low schooling and $29.4 \%$ with a high nicotine dependence. No significant differences were found in the association between demographic variables and the level of dependence. Conclusion: The high level of nicotine dependence in individuals who attend the program may help actions for smoking cessation favoring strategies to increase participation in smoking control groups.

DESCRIPTORS: Smoking Prevention; Smoking; Tobacco Use Disorder.

\section{RESUMEN}

Objetivo: Identificar la dependencia de la nicotina en usuarios que no han dejado de fumar después del Programa de Control del Tabaco en la unidad de salud Gold Seal. Método: Estudio de caso cuantitativo, realizado en la Unidad Básica de Salud Sello de Oro de Maringá-PR, en 2020. Participaron participantes que asistieron a todas las sesiones del programa en 2018 y 2019, y que no dejaron de fumar luego de la intervención. Para la recolección, utilizamos entrevistas guiadas por un instrumento estructurado. Los datos se organizaron utilizando Microsoft Excel 2016 y se analizaron estadísticas descriptivamente simples. Respetando todos los preceptos éticos. Resultados: Hubo 17 participantes, 64,7\% mujeres, 58,8\% mayores de 60 años, 35,3\% casados, 47,05\% con baja escolaridad y $29,4 \%$ con alta dependencia a la nicotina. No hubo significación asociativa entre las variables demográficas y el grado de dependencia. Conclusión: El alto grado de dependencia de la nicotina en los participantes del programa puede ayudar a las acciones para dejar de fumar al favorecer estrategias para ampliar la participación en los grupos de control del tabaco.

DESCRIPTORES: Prevención del tabaquismo; De fumar; Abandono del consumo de tabaco.

RECEBIDO EM: 08/04/2021 APROVADO EM: 09/04/2021

\section{Camila Salci Capelasso}

Acadêmico do Curso de Medicina, UNICESUMAR, Maringá-PR.

ORCID: 0000-0003-4893-0277 


\section{Laís Loidi Garbugio}

Acadêmico do Curso de Medicina, UNICESUMAR, Maringá-PR.

ORCID: 0000-0001-5621-7813

\section{Francielle Renata Danielli Martins Marques}

Doutoranda do Programa de Pós-graduação em Enfermagem, Universidade Estadual de Maringá, Maringá-PR.

ORCID: 0000-0002-8578-9615

\section{Graziele Adrieli Rodrigues Pires}

Doutoranda do Programa de Pós-graduação em Enfermagem, Universidade Estadual de Maringá, Maringá-PR.

ORCID: 0000-0002-9673-9218

\section{Maria Aparecida Salci}

Doutora. Docente do Programa de Pós-graduação em Enfermagem, Universidade Estadual de Maringá, Maringá-PR.

ORCID: 0000-0002-6386-1962

\section{Patricia Bossolani Charlo}

Orientadora, Doutoranda, Docente do Curso de Enfermagem e Medicina na UNICESUMAR, Maringá-PR.

ORCID: 0000-0002-8262-2086

\section{INTRODUÇÃO}

0 tabaco é considerado um dos principais fatores de mortalidade por causas evitáveis no mundo, com cerca de cinco milhões de óbitos anualmente $^{1}$. O uso do tabaco teve uma considerável influência midiática com grandes artistas renomados incitando a adesão ao cigarro no fim do século 20. Com a identificação dos malefícios causados pelo consumo do produto, foram criadas leis que regulamentaram o programa de controle do tabagismo a fim de suprimir a demanda crescente do consumo do tabaco.

No Brasil, com o intuito de reduzir a utilização do tabaco, foi implementada a Lei n. 9.294, de 15 de julho de 1996, com a suspensão das propagandas comerciais em todo território nacional que abordassem cigarros, charutos ou qualquer produto fumígeno derivado ou não do tabaco ${ }^{2}$.

Como resultado foi possível visualizar a redução do tabagismo entre 2006 a 2019 , com queda de $15,7 \%$ para $9,8 \%$ do consumo em pessoas maiores de 18 anos $^{3}$. Além disso, foram observadas que as tentativas de cessar o consumo do tabaco aumentaram de $41,3 \%$ para $47,2 \%$. Na análise por regiões brasileiras, as regiões sul e sudeste concentram as maiores taxas de usuários, $16,1 \%$ e $15 \%$, respectivamente. Em relação ao sexo, os homens são os maiores consumidores $(12,3 \%)^{2}$.
A expressiva redução está atrelada ao Tratado da Convenção-quadro para Controle do Tabaco (CQCT), firmado entre diversos países, a fim de adotarem novas e rigorosas medidas políticas, sob a fiscalização e controle da Organização Mundial de Saúde, que fornece as diretrizes para implementação de programas de combate ao tabagismo ${ }^{3}$.

A implantação da CQCT no Brasil, possibilitou a integração do Programa Nacional de Controle do Tabagismo (PNCT) à Política Nacional de Controle do Tabaco do Estado Brasileiro, que dispõe da utilização dos profissionais da área da saúde, especificamente a Atenção Primária a Saúde (APS), na linha de frente de acordo com a identificação das necessidades locais. Dessa forma, forneceu autonomia aos municípios para implantação de sessões semanais, quinzenais ou mensais, com base na demanda espontânea, nível de dependência, e número de participantes integrados ao programa ${ }^{3,4}$.

A dependência a nicotina é um dos principais fatores que dificultam o processo de abandono ou cessação do consumo do tabaco, já que esta é uma substância psicoativa que age no sistema nervoso causando alterações comportamentais e emocionais à pessoa, resultando em uma compulsão pelo uso dessa substância4. Dessa forma, é indispensável as ações do Programa de Controle do Tabagismo (PCT), composto por equipe interdisci- plinar, realizado pela Atenção Primária à Saúde (APS) para coordenar e promover uma atenção integral que viabilize a cessação tabágica, resultando na melhoria das condições de saúde das pessoas 5 .

Os níveis de dependência à nicotina podem influenciar no grau de motivação e contemplação para o processo de cessação, pois há associação do maior nível de dependência com os altos índices de recaídas6. Para avaliar e identificar o nível de dependência foi elaborado o Teste de Fagerstrom, validado no Brasil por Carmo \& Pueyo a partir de uma adaptação em 19916. Este teste consiste em seis escalas com escores de 0 a 3 que formam um total de 10 pontos, sendo os escores de 0 a 2 considerados dependência de grau muito baixo; 3 a 4, grau baixo; 5, grau médio; 6 a 7, elevados; e 8 a 10, muito elevado7.

Diante dessas considerações, emergiu a seguinte pergunta de pesquisa: Qual o grau de dependência a nicotina, segundo o Teste de Fagerstrom, das pessoas que finalizaram a participação nos grupos do PCT de uma Unidade Básica de Saúde (UBS) selo ouro e que não cessaram o consumo de tabaco?

Sendo assim, o objetivo deste estudo foi identificar o grau de dependência à nicotina em usuários que não cessaram o consumo de tabaco após participar do PCT em uma unidade de saúde classificada como selo ouro. 


\section{MÉTODO}

Trata-se de um estudo de caso de natureza quantitativa, realizado em uma UBS selo ouro, localizada em um município de médio porte situado no noroeste do Paraná, que consta com um total de 34 UBS, sendo duas classificadas e certificadas com o selo ouro e onze com selo bronze. Um dos requisitos imprescindíveis para a garantia e manutenção do selo ouro, conforme o projeto QualificaAPSUS são os resultados/ações para comunidade. Contudo, em 2018 e 2019, somente uma UBS desenvolveu o PCT e em 2020, devido a pandemia por COVID-19, as atividades grupais foram suspensas.

A UBS inaugurada em 2013 atende uma população de aproximadamente seis mil habitantes e contém uma equipe da Estratégia Saúde da Família (ESF) coordenada por uma enfermeira e responsável pela condução do PCT. A equipe de saúde desta unidade trabalha com a interdisciplinaridade, possibilitando a manutenção do selo ouro e a efetividade no atendimento ao usuário cadastrado na área de abrangência.

A UBS, realiza as atividades do PCT, na qual os profissionais das áreas de enfermagem, psicologia, medicina e nutrição, oferece terapia cognitivo-comportamental e tratamento farmacológico combinado quando necessário, conduzidos por dois modelos de sessões periódicas, com duração de uma hora cada, que priorizam o atendimento em grupo de apoio à cessação tabagista e/ou individual nos casos de impossibilidade da oferta dos grupos. Esses grupos são organizados em quatro sessões semanais e, para os que conseguiram cessar o consumo do tabaco, uma a duas sessões quinzenais de manutenção da abstinência, objetivando conhecer as dificuldades de cada pessoa para permanecer sem fumar e auxiliá-las conforme necessário. E, uma sessão mensal aberta, para prevenção de recaída, até completar um ano, com orientações para o fortalecimento da decisão de permanecerem abstinentes.

Os participantes do estudo foram usuários que compareceram em todas as sessões do PCT em 2018 e 2019, e que não
A UBS inaugurada em 2013 atende uma população de aproximadamente seis mil habitantes e contém uma equipe da Estratégia Saúde da Família (ESF) coordenada por uma enfermeira e responsável pela condução do PCT. A equipe de saúde desta unidade trabalha com a interdisciplinaridade, possibilitando a manutenção do selo ouro e a efetividade

no atendimento ao usuário cadastrado na área de abrangência. cessaram o consumo de tabaco após a intervenção. Foram excluídas as pessoas com idade inferior a 18 anos.

A coleta de dados ocorreu no período de março a maio de 2020 utilizando como técnica a entrevista, realizada de modo virtual devido o avanço da pandemia por Covid-19 no Brasil e as orientações de distanciamento social. As entrevistas foram guiadas por um instrumento estruturado, subdividido em duas sessões: a primeira versava sobre a caracterização sócio-demográfica; e a segunda, a aplicação do Teste de Fagerstrom para identificar o grau de dependência do tabaco nos participantes, mesmo após a intervenção do PCT.

Para organização dos dados, foi utilizado o Software Microsoft Excel 2016. A análise foi por meio de estatística descritiva simples, com a frequência relativa e absoluta. O teste exato de Fisher, realizado pelo site de OpenEpi e com nível de significância de 5\%, foi utilizado para verificar a associação entre o grau de dependência com as condições sócio demográficas dos participantes.

Todos os preceitos éticos foram respeitados, conforme resoluções 466/2012 e 510/2016 do Conselho Nacional de Saúde, obtendo autorização da Secretaria de Saúde Municipal e aprovação do COPEP (Comitê Permanente de Ética em Pesquisa com Seres Humanos) sob parecer $\mathrm{n}^{0} 2.278 .656$ de 15/09/2017 e CAAE: 57222016.1.0000.0104. Todos os participantes da pesquisa assinaram ou concordaram verbalmente com o Termo de Consentimento Livre e Esclarecido (TCLE).

\section{RESULTADOS}

Em 2018 e 2019, participaram do PCT 18 pessoas. Entretanto, em 2018, mesmo após o processo de intervenção e a conclusão das sessões, nenhum participante aderiu aos objetivos do PCT de cessação tabágica. Contudo, em 2019, uma pessoa alcançou o objetivo de cessação tabágica mesmo com grau de dependência a nicotina elevado.

Dessa forma, o presente estudo obteve uma amostra de 17 participantes, sendo: 
TABELA 1. Caracterização e grau de dependência dos participantes do PCT. Maringá, PR, 2021

\begin{tabular}{|c|c|c|}
\hline VARIÁVEIS & $\mathbf{N}$ & $\%$ \\
\hline \multicolumn{3}{|l|}{ Sexo } \\
\hline Feminino & 11 & 64,7 \\
\hline Masculino & 6 & 35,3 \\
\hline \multicolumn{3}{|l|}{ Idade } \\
\hline 30-39 & 1 & 5,9 \\
\hline $40-49$ & 5 & 29,4 \\
\hline $50-59$ & 1 & 5,9 \\
\hline $60-69$ & 10 & 58,8 \\
\hline \multicolumn{3}{|l|}{ Estado civil } \\
\hline Solteiro (a) & 3 & 17,65 \\
\hline Casado (a) & 6 & 35,3 \\
\hline Divorciado (a) & 5 & 29,4 \\
\hline Viúvo (a) & 3 & 17,65 \\
\hline \multicolumn{3}{|l|}{ Escolaridade } \\
\hline $1^{\circ}$ grau incompleto & 8 & 47,05 \\
\hline $1^{\circ}$ grau completo & 3 & 17,65 \\
\hline $2^{\circ}$ grau incompleto & 1 & 5,9 \\
\hline $2^{\circ}$ grau completo & 5 & 29,4 \\
\hline Nível superior & 0 & 0 \\
\hline \multicolumn{3}{|l|}{ Grau de Dependência } \\
\hline Muito Baixo (0-2) & 1 & 5,9 \\
\hline Baixo (3-4) & 1 & 5,9 \\
\hline Médio (5) & 6 & 35,3 \\
\hline Elevado (6-7) & 5 & 29,4 \\
\hline Muito Elevado (8-10) & 4 & 23,5 \\
\hline squisadores, 2020 & & \\
\hline
\end{tabular}

Fonte: dados dos pesquisadores, 2020

\section{TABELA 2. Análise das variáveis relacionadas ao grau de dependência ao} tabaco, Maringá, 2020

$\begin{array}{ccccc}\begin{array}{c}\text { VARIÁVEIS PERFIL } \\ \text { Sexo }\end{array} & \text { DEPENDÊNCIA } \leq 5 & \text { DEPENDÊNCIA }>5 & \text { TOTAL } & P \\ \begin{array}{c}\text { Feminino } \\ \text { Masculino }\end{array} & 5 & 6 & 11 & 0.99 \\ \text { Idade } & 3 & 3 & 6 & \\ <60 \text { anos } & 3 & 4 & 7 & 0.99 \\ >60 \text { anos } & 5 & 5 & 10 & \\ \text { Estado Civil } & & & 10 & 0.99 \\ \text { Não casado } & 5 & 5 & 7 & \\ \text { Casado } & 3 & 4 & & \end{array}$

$11(64,7 \%)$ do sexo feminino; com predomínio de idosos $(58,8 \%)$, casados (35,3\%), com baixa escolaridade (47,05\%), grau de dependência a nicotina variando de baixo a muito elevado com predominância de índices médio e muito elevado, conforme Tabela 1.

O grau de dependência a nicotina foi avaliado de acordo com o Teste de Fagestrom, que pontua a resposta dos participantes em seis questões objetivas. A primeira envolve o tempo para acender o primeiro cigarro no dia, na qual a maioria refere que isso ocorre nos primeiros cinco minutos do dia, logo ao acordar. Quando questionado sobre segunda questão, referente à dificuldade de não fumar em ambientes coletivos, relacionado às leis vigentes nas três esferas governamentais, foram unânimes as queixas expressando a dificuldade em manter a abstinência nesses ambientes.

A terceira questão se refere ao cigarro que apresenta a maior satisfação ao ser consumido, não havendo consenso entre as respostas em relação ao primeiro ou aos demais cigarros do dia. A quarta questão se refere ao número de cigarros consumidos em 24 horas, revelando índices alarmantes e correlacionados ao grau de dependência, pois, sete participantes consomem de 11 a 20 cigarros/dia, enquanto que cinco consomem acima de 21 cigarros/dia.

Com relação ao período dia em que consomem o maior número de cigarros, os períodos vespertinos e noturnos obtiveram maiores índices. A última questão está associada ao processo de cessação com o adoecimento, na qual os participantes referiram interromperem o consumo do tabaco ao serem acometidos por alguma patologia, retornando assim que o quadro patológico melhore ou estabilize.

O teste exato de Fisher, utilizado para verificar a associação entre as variáveis demográficas e o grau de dependência, não apresentou diferenças significativas, tanto para o sexo, idade ou escolaridade. Este fato pode estar associado ao baixo número de participantes no PCT, o que dificulta a avaliação estatística (erro tipo II), conforme Tabela 2. 

Inferior a 10 anos
5
6
Superior a 10 anos
4
2
11
0.75

Fonte: dados dos pesquisadores, 2020

\section{DISCUSSÃO}

O sexo feminino obtém a maior procura espontânea por programas e serviços ofertados pelas unidades de saúde, pela busca de melhores condições de vida e saúde. A predominância do público feminino na procura dos PCT está associa$\mathrm{da}$ aos fatores intervenientes do processo de cessação, como a relação com o prazer, alívio de sentimentos negativos, situações estressantes e ganho de peso $^{8}$.

De acordo com o último censo do Programa Nacional de Saúde (PNS) (2019), observa-se maior taxa de fumantes entre os 40 aos 59 anos de idade. Entretanto, os resultados deste estudo demonstram que os idosos são os que mais procuraram auxílio no PCT para cessação do vício?. Três aspectos estão correlacionados a essa procura por pessoas acima dos 60 anos: maior conscientização sobre os malefícios do consumo de tabaco; maior proximidade de pessoas com complicações associadas ao hábito de fumar; por pedido dos familiares e ou indicação dos profissionais de saúde, sendo esta a principal motivação com relevância para o sucesso do tratamento ${ }^{10}$.

O estado civil casado foi predominante, já que o apoio entre o casal pode facilitar o abandono do uso de tabaco. Há também uma grande procura do PCT por pessoas divorciadas, que apresentam maior dificuldade com a cessação pois, estão em uma situação de maior fragilidade ${ }^{11}$.

Um fator de risco para o consumo de tabaco identificado na pesquisa é a baixa escolaridade, o que pode ser decorrente da predominância de participantes com idade acima de 60 anos, representando uma proporção significativa de brasileiros com baixa escolaridade ou com menos de um ano de estudo. Essa relação é importante, pois, a iniciação do consumo de tabaco está associada com o pico de abandono dos estudos entre 17 e 19 anos $^{6}$.
O sexo feminino

obtém a maior

procura espontânea

por programas e

serviços ofertados

pelas unidades de

saúde, pela busca de

melhores condiçôes

de vida e saúde. A

predominância do

público feminino

na procura dos

PCT está associada

aos fatores

intervenientes do

processo de cessação,

como a relação

com o prazer, alívio

de sentimentos

negativos, situações

estressantes e ganho

de peso.
No Brasil, pessoas com baixo grau de escolaridade têm probabilidade 35 vezes maiores de serem fumantes quando comparadas às pessoas que possuem o segundo grau completo ou nível superior ${ }^{6}$. Um estudo de coorte no Rio Grande do Sul, realizado com adolescentes acompanhados desde o seu nascimento revelou que o tabagismo foi associado inversamente a escolaridade ${ }^{12}$.

O grau de dependência apresentado na presente pesquisa oscila entre médio e muito elevado, corroborando com pesquisa realizada em um município próximo ao da pesquisa, no qual houve a prevalência de altas taxas de dependência a nicotina e a predominância de mulheres na participação em PCT, visto que esse público apresenta maiores dificuldades para interromper o uso da nicotina sem auxílio profissional, em virtude da associação da dependência química, psicológica e comportamental, contrapondo-se ao sexo masculino que, mesmo apresentando um maior grau de dependência a nicotina, possui sucesso na cessação tabágica após intervenção medicamentosa ${ }^{13}$.

Ao analisar individualmente o teste de Fagerstrom, as primeiras três questões apresentam um valor preditivo para o resultado, pois, estão relacionadas com o grau de satisfação do consumo, associado aos receptores neuronais em que a nicotina faz sua ligação, ocorrendo a liberação excessiva de dopamina e endorfinas; quando esse estímulo se dissipa, os receptores sinalizam uma necessidade de um novo estímulo (mais nicotina), isso em fumantes regulares tem efeitos cotidianos, aparecendo sinais de abstinências em curtos períodos em que fiquem impedidos de fumar ${ }^{2,14}$.

Esse hábito se caracteriza como dependência comportamental por associar o tabaco com hábitos diários de sua rotina, como o despertar. Quanto maior o hábito de fumar nas primeiras horas do dia, maior é o grau de dependência do paciente ${ }^{10}$.

Neste estudo, não houve correlação significativa entre o grau de dependência e as variáveis pesquisadas, o que demonstra dificuldades no processo de cessação 
tabágica, já que esse fenômeno envolve um olhar ampliado para a sua compreensão e um detalhamento sobre as diversas abordagens individuais da tríade paciente, unidade de saúde e condicionantes/ determinantes de saúde ${ }^{15,16}$.

Em um estudo em que foram utilizadas técnicas de mapeamento em saúde nas Unidades Básicas de Saúde, demonstrou que $59,1 \%$ dos usuários dos Programas de Atendimento a Hipertensos e Diabéticos Hiperdia eram tabagistas ${ }^{17}$.

\section{CONCLUSÃO}

Com base no presente estudo, conclui-se que a UBS apresenta a predominância de procura ao PCT pelo público feminino, acima de 50 anos, com baixa escolaridade e um grau de dependência alto, o que dificulta o processo de cessação tabágica. Este resultado indica a necessidade de estratégias que auxiliem as pessoas e tornem possível a cessação tabágica, principalmente para àquelas com grau de dependência elevado.
Os resultados apontaram a necessidade de fortalecer a implementação de novas estratégias e metas a serem alcançadas por meio das unidades de saúde, almejando uma procura mais expressiva de participantes, principalmente nas unidades que almejam conquistar ou garantir a permanência do selo ouro.

Dessa forma, novas pesquisas devem ser incentivadas, proporcionando a compreensão multifatorial do tabagismo, a fim de viabilizar a cessação tabágica e auxiliar as UBS na condução do PCT por equipes interdisciplinares.

\section{REFERÊNCIAS}

1. Amorim TA, Lucchese R, Silva NEM, Santos JS, Vera I, Paula NI et al . Determinantes de saúde mental e abuso de substâncias psicoativas associadas ao tabagismo. Estudo de caso controle. Ciênc. saúde coletiva. 2019;24(11): 4141-4152.

2. Oliveira RM, Santos JLF, Furegato ARF. Prevalência e perfil de fumantes: comparações na população psiquiátrica e na população geral. Rev. Latino-Am. Enfermagem. 2019;27: e3149.

3. Pinto M, Bardach A, Palacios A, Biz A, Alcaraz A, Rodriguez $B$, et al . Carga do tabagismo no Brasil e benefício potencial do aumento de impostos sobre os cigarros para a economia e para a redução de mortes e adoecimento. Cad. Saúde Pública. 2019;35(8):e00129118.

4. Zanini R, Sotili M. Uso de drogas, repercussões e intervenções neuropsicológicas em saúde mental. Cadernos Brasileiros de Saúde Mental/Brazilian Journal of Mental Health, 11, jun. 2019.

5. Portes LH, Campos EMS, Teixeira MTB, Caetano R, Ribeiro LC. Ações voltadas para o tabagismo: análise de sua implementação na Atenção Primária à Saúde. Ciênc. saúde coletiva. 2014;19(2):439-448.

6. Leal ACS et al. Características sociodemográficas e padrão de consumo tabagístico de fumantes que procuram atendimento em programas de cessação. Mundo saúde (Impr.), 163-169, 2017.

6. Pietrobon RC; Barbisan JN; Manfroi W. Uso do teste de Fagerström para dependência de nicotina como um instrumento para medir a dependência de nicotina. Clinical \& Biomedical Research. 2007; 27(3).

7. Arendartchuk D.; Ayala ALM. Fatores associados à cessação do tabagismo entre participantes de um programa antitabagista em uma Unidade Básica de Saúde de Joinville - SC. Revista de APS. 2020; 21(4):1-8.

8. Mattos, LR et al. Cessação do tabagismo entre usuários da Estratégia Saúde da Família [Cessation of smoking among Family Health Strategy users] [Cese del tabaquismo entre usuarios de la Estrategia de Salud Familiar]. Revista Enfermagem UERJ. 2019; 27:e38987.
9. Brasil. Ministério da Saúde (BR). Secretaria de Vigilância em Saúde. Departamento de Análise em Saúde e Vigilância de Doenças Não Transmissíveis. Vigitel Brasil 2019: vigilância de fatores de risco e proteção para doenças crônicas por inquérito telefônico. Brasília: Ministério da Saúde; 2020, 137 p.

10. Jesus MCP, Silva MH, Cordeiro SM, Kortchmar E, Zampier VSB, Merighi MAB. Understanding unsuccessful attempts to quit smoking: a social phenomenology approach. Rev. esc. enferm. USP. 2016;50( 1 ):71-78.

11. Müller EV et al. Fatores associados ao tabagismo em usuários da estratégia saúde da família. Cogitare Enfermagem, 2017;22(4). ISSN 2176-9133.

12. Menezes AMB, Hallal PC, Horta BL. Determinantes precoces do tabagismo na adolescência: um estudo prospectivo de coorte de nascimentos. Cafajeste. Saúde Pública 2007; 23 (2): 347-354.

13. Oliveira GMM, Mendes M, Dutra OP, Achutt A, Fernandes M, Azevedo V, et al. Recomendações para a Redução do Consumo de Tabaco nos Países de Língua Portuguesa - Posicionamento da Federação das Sociedades de Cardiologia de Língua Portuguesa. Arq. Bras. Cardiol. 2019;112( 4 ): 477-486.

14. Halty LS, Hüttner MD, Oliveira NIC, Santos VA, Martins G. Análise da utilização do Questionário de Tolerância de Fagerström (QTF) como instrumento de medida da dependência nicotínica. J. Pneumologia. 2002;28( 4 ): 180-186.

15. Amsterdan JV. Use of the Fagerström test to assess diferences in the degree of nicotine dependence in smokers from five ethnic groups: The helius study. Drug and Alcohol Dependence, 2019;194:197-204.

16. Guibu IA, Moraes JC, Guerra JAA, Costa EA, Acurcio FA, Costa KS et al . Características principais dos usuários dos serviços de atenção primária à saúde no Brasil. Rev. Saúde Pública. 2017;51(Suppl 2):17s.

17. Santos MG dos, Christovam BP, Lima VM da F. Mapeamento em saúde como ferramenta para gerência do cuidado aos idosos hipertensos na atenção primária. SaudColetiv (Barueri). 2021;9(49):1509-1512. 